\title{
Análise bioquímica da saliva de pacientes com deficiência cognitiva e física como estratégia de aula prática de Bioquímica no curso de Odontologia
}

\author{
Antonio Hernandes Chaves Neto*; Maria Flávia Marques Ribeiro**; Ana Cláudia de Melo Stevanato \\ Nakamune* \\ * Professor (a) Assistente Doutor (a), Universidade \\ Estadual Paulista (UNESP), Faculdade de Odontologia \\ de Araçatuba (FOA), Departamento de Ciências \\ Básicas, Câmpus de Araçatuba e Programa de Pós- \\ Graduação Multicêntrico em Ciências Fisiológicas, \\ UNESP-SBFis \\ ** Professora Titular, Universidade Federal do Rio Grande \\ do Sul (UFRGS), Instituto de Ciências Básicas da Saúde \\ (ICBS), Departamento de Fisiologia
}

Recebido em 09/03/2020. Aprovado em 27/08/2020.

\begin{abstract}
RESUMO
Diante da importância do ensino de Bioquímica na formação do futuro cirurgião-dentista, o objetivo é apresentar uma estratégia pedagógica que possibilita a articulação dos conceitos moleculares básicos da disciplina no entendimento da complexidade das doenças bucais e sistêmicas, por meio da análise bioquímica da saliva de pacientes com deficiência cognitiva e física do Centro de Assistência Odontológica à Pessoa com Deficiência (CAOE) da Faculdade de Odontologia de AraçatubaUniversidade Estadual Paulista (UNESP). Para tanto, grupos de cinco estudantes realizaram as análises na saliva, coletada por um cirurgião-dentista do CAOE. Imediatamente após a coleta, foi determinado o fluxo salivar, $\mathrm{pH}$ e a capacidade tamponante. Na sequência, a saliva foi centrifugada, fracionada e armazenada a $-20^{\circ} \mathrm{C}$ até o momento das análises bioquímicas. Durante as aulas práticas, realizaram-se os seguintes ensaios, utilizando kits comerciais: proteína total, $\alpha$-amilase, fosfatase alcalina, ácido úrico, glicose, aspartato aminotransferase, cálcio e fósforo. Ao final do ano letivo, os estudantes apresentaram relatório contextualizando os resultados com a saúde bucal e sistêmica do paciente. Plantões de dúvidas com monitores e professores auxiliaram na interpretação da ficha de anamnese e nas correlações dos parâmetros clínicos bucais e sistêmicos. Para os estudantes ingressantes, foi a primeira oportunidade de integrar o conhecimento teórico às condições clínicas de um paciente. Conclui-se que a estratégia adotada é viável e pode beneficiar educadores que buscam alternativas que permitam a integração das ciências básicas e clínicas ao ensino de Bioquímica para a Odontologia.
\end{abstract}

Descritores: Aprendizagem. Ensino. Educação em Odontologia. Bioquímica. Saliva. 


\section{INTRODUÇÃO}

A saliva tem sido muito utilizada como material biológico em testes rápidos e sensíveis, que possibilitam o diagnóstico precoce e o monitoramento de doenças ${ }^{1}$. Por mais que esse cenário evidencie a importância do ensino de Bioquímica para os discentes do curso de Odontologia, muitos ainda encontram dificuldades no processo de aprendizado e contextualização da disciplina $^{2-4}$. Os estudantes atribuíram dita dificuldade à carência de práticas que demonstrem a relevância dos conteúdos ministrados e, por esse motivo, não é claro para eles que é nas aulas de Bioquímica que lhes são apresentadas as bases moleculares que justificam a necessidade de uma boa higiene dental ${ }^{3}$.

O ensino baseado em casos clínicos já se mostrou um facilitador eficaz no processo de aprendizagem de muitas disciplinas, incluindo a Bioquímica, além de melhorar o interesse do estudante $^{5}$, fazendo com que ele se sinta mais motivado a participar como membro ativo da sala de aula, ajudando na tomada de decisões e trabalhando com o grupo durante as discussões acerca da prática clínica. Ademais, já foi provado que esse tipo de experiência incentiva o discente a desenvolver habilidades fundamentais para $\mathrm{o}$ aprendizado ao longo da vida ${ }^{6}$. Esta metodologia de ensino envolve ativamente o estudante, expondo-o ao processo científico através do estudo de casos reais ${ }^{7}$, fomentando o uso de informações na resolução de problemas e facilitando a retenção do conhecimento e o aprimoramento da comunicação ${ }^{8}$.

As Diretrizes Curriculares Nacionais (DCN) para o curso de graduação em Odontologia no Brasil foram instituídas em 19 de fevereiro de 2002, por meio da resolução CNE/CES 3, definindo princípios, fundamentos, condições e procedimentos necessários para a formação de cirurgiões-dentistas no Brasil. Elas determinam que a estrutura do curso de Odontologia deverá utilizar metodologias de ensino/aprendizagem que permitam o desempenho de um papel ativo neste processo por parte do estudante, em adição à integração dos conhecimentos das ciências básicas aos das ciências clínicas ${ }^{9}$. Dentro da formação básica do curso de Odontologia, destaca-se o conjunto de conhecimentos que compreendem as Ciências Fisiológicas: Bioquímica, Fisiologia e Farmacologia ${ }^{9}$. A intenção do domínio de ciências básicas é que o estudante adquira embasamento científico necessário para o tratamento clínico dos pacientes e que ele desenvolva sua capacidade de pensamento e análise de dados ${ }^{10}$.

Transformar estudantes em profissionais competentes e atenciosos, capazes de melhorar os cuidados de saúde bucal de uma sociedade, é um dos principais objetivos da educação odontológica $^{11}$ e um desafio considerável para os educadores da área. Para os discentes desse curso, o ciclo de aprendizagem também é marcado por contratempos, pois o processo de contextualização dos conhecimentos teóricos integrados à prática, aprendendo sobre as patologias e enriquecendo suas habilidades ${ }^{12}$, por vezes apresenta adversidades e nem todos o executam com tranquilidade.

Por conta dos desafios que pontuam esse processo de ensino-aprendizagem, tanto para educadores como para educandos, são propostas diversas estratégias de auxílio para uma melhor aquisição de conhecimentos e para que a comunicação dos conteúdos pelo professor seja facilitada. $\mathrm{Na}$ área de Odontologia, já foi constatado que o modelo tradicional de aulas expositivas apresenta várias limitações, pois a comunicação unidirecional com falta de interação resulta em baixo envolvimento dos estudantes ${ }^{13}$. Neste contexto, um estudo sobre ensino de Fisiologia Humana mostrou que a discussão de casos clínicos na área básica é uma ferramenta importante para melhorar o aprendizado e o desempenho dos estudantes ${ }^{14}$. 
$\mathrm{O}$ atendimento odontológico a pacientes portadores de necessidades especiais é diferenciado, uma vez que estes apresentam alterações físicas e/ou mentais que podem ser temporárias ou permanentes ${ }^{15}$. Em nossa unidade universitária, a possibilidade de trabalhar com a saliva desses pacientes se mostrou viável, em função da existência do Centro de Assistência Odontológica à Pessoa com Deficiência (CAOE), que presta atendimento à essa parcela da população.

Diante da importância do ensino de Bioquímica na formação do futuro cirurgiãodentista, o objetivo desta estratégia pedagógica é possibilitar a articulação dos conceitos moleculares básicos e clínicos da disciplina no entendimento da complexidade das doenças bucais e sistêmicas, por meio da análise bioquímica da saliva de pacientes com deficiências cognitiva e física.

\section{RELATO DE EXPERIÊNCIA}

Trata este artigo de um relato descritivo de uma experiência aplicada aos estudantes do $1^{\circ}$ ano dos cursos de Odontologia Integral e Noturno da FOA-UNESP. A ideia é fruto das discussões alavancadas pela dificuldade encontrada por estudantes e professores da disciplina de Bioquímica no processo de ensino-aprendizagem, que pode estar relacionada à complexidade inerente ao conteúdo, à sua elevada abstração e à ampla gama de origens das informações apresentadas ${ }^{16,17}$.

A disciplina é anual, obrigatória e contempla os conteúdos de Bioquímica Estrutural, Metabólica e Bucal. A carga horária semanal é de 3 horas aula/turma perfazendo um total anual de 90 horas/turma. Para que os conteúdos básico e bucal sejam mais bem integrados, os assuntos foram agrupados por temas afins, viabilizando o estabelecimento de correlações entre teoria e prática, e permitindo que fossem alcançados os objetivos pedagógicos. A sequência cronológica das atividades pode ser observada na tabela 1 .
As estratégias pedagógicas adotadas no ano de 2019 foram aulas teóricas expositivas com auxílio de projetor multimídia e quadro branco, aulas práticas laboratoriais e discussão dos dados obtidos nas mesmas. As aulas teóricas forneciam embasamento para uma abordagem interpretativa dos resultados das análises obtidos nas aulas práticas. Esse conhecimento prévio foi de grande importância para o melhor aproveitamento das aulas práticas por parte do estudante ${ }^{18}$. Para as práticas, eram disponibilizados aos estudantes, com antecedência, os guias de aula, contendo uma breve introdução que resgatava conceitos abordados na teoria, além das instruções e descrição das etapas para o desenvolvimento das análises bioquímicas salivares.

Para as atividades práticas, cada turma foi subdivida em grupos de cinco estudantes, escolhidos entre si por afinidade e mantidos até o final do ano letivo. Nas duas primeiras aulas, foi abordada a biossegurança, o reconhecimento e a utilização correta de materiais de laboratório. Foram ensinadas e praticadas técnicas corretas para utilização de pipetas graduadas de vidro e pipetas automáticas. Nas semanas posteriores, três horas/aula ficaram reservadas para o treinamento da coleta de saliva de um estudante voluntário de cada grupo. Nesta mesma oportunidade, os estudantes aprenderam os procedimentos para a determinação do fluxo salivar, $\mathrm{pH}$ e capacidade tamponante, e finalizaram centrifugando a saliva e fracionando o sobrenadante para armazenamento. Dessa forma, os estudantes foram preparados para o trabalho póstero com a saliva dos pacientes do CAOE.

Em data e horário agendados para cada turma (A, B e Noturno), uma palestra no anfiteatro do CAOE foi ministrada pela coordenação, para apresentar a estrutura da unidade auxiliar, sua logística e as peculiaridades para o atendimento dos pacientes com necessidades especiais. $\mathrm{Na}$ sequência, dois componentes aleatórios de cada 
grupo, devidamente paramentados e com uma ficha de anamnese simplificada fornecida pela disciplina de Bioquímica, foram encaminhados, aos consultórios odontológicos do Centro.

A coleta de saliva foi realizada com Salivette ${ }^{\circledR}$ pelo profissional da odontologia que atendia rotineiramente o paciente, para evitar expôlo às situações que pudessem gerar estresse. Os roletes de algodão estéril foram posicionados no assoalho da boca por no máximo cinco minutos e, em seguida, transferidos ao laboratório nos Salivette ${ }^{\circledR}$, mantidos em banho de gelo. Foram então estimados fluxo salivar, valor de $\mathrm{pH}$ e capacidade tamponante. É importante observar que todos os pacientes envolvidos apresentam comprometimento cognitivo e/ou físico que exige atendimento especializado, mas por diferentes causas. Transtorno do espectro do autismo, síndrome de Down e paralisia cerebral foram as causas predominantes.

No momento da coleta da saliva, os estudantes puderam anotar nas fichas de anamnese da disciplina o nome e o número de prontuário CAOE do paciente. Posteriormente, foram instruídos a finalizar o preenchimento da ficha consultando o prontuário do Centro, extraindo as seguintes informações: nome, idade, gênero, cor, tipo de alimentação, hábitos bucais, motivo da consulta, medicações em uso e doenças pregressas. Informações sobre a presença de respiração bucal, condições de higiene bucal, dependência para a higienização bucal, presença ou não de alterações no fluxo salivar (sialorreia, xerostomia), características da língua, condição periodontal e odontograma foram também transcritas para a versão simplificada do prontuário. $\mathrm{O}$ número elevado de estudantes em cada turma exigiu de toda a equipe envolvida uma logística bem estruturada e organizada. Durante a coleta das amostras de saliva, os estudantes da turma que não foram aos consultórios assistiram ao procedimento por transmissão simultânea, acompanhados de um professor da disciplina, para esclarecimentos.

Após as determinações de fluxo salivar, $\mathrm{pH} \mathrm{e}$ capacidade tamponante, as amostras de saliva foram centrifugadas, fracionadas e armazenadas em frascos cônicos de 0,5 mL, contendo os volumes necessários para a determinação, em semanas distribuídas ao longo do ano letivo, dos seguintes parâmetros: proteína total, $\alpha$-amilase, fosfatase alcalina, glicose, ácido úrico, aspartato aminotransferase, cálcio e fósforo. As alíquotas devidamente identificadas com o número do grupo e turma foram armazenadas na temperatura de -20 ${ }^{\circ} \mathrm{C}$. Nenhuma alíquota foi descongelada mais de uma vez.

Após a análise do parâmetro salivar, os resultados obtidos pelos grupos eram transcritos para o quadro branco nas aulas práticas. Nesse momento, cada grupo era indagado sobre as condições clínicas bucais e sistêmicas dos pacientes, e cabia ao docente assumir postura questionadora e crítica, encorajando os estudantes na busca por informações na literatura que permitissem o estabelecimento de correlações entre os resultados obtidos e os dados clínicos do paciente. Dessa forma, fomentava-se a reflexão depois da ação. Após a discussão inicial, os estudantes eram impelidos novamente à ação, validando suas reflexões por meio da pesquisa de trabalhos em bases de dados como SciELO e PubMed. O desenvolvimento do relatório, entregue pelo grupo ao final do ano letivo, permitiu aperfeiçoar a habilidade de escrever um relatório técnico-científico.

Para a atribuição de nota ao relatório, computada na média final da disciplina, foram utilizados os seguintes critérios: formatação do trabalho e qualidade da apresentação gráfica; obediência às normas para citação das referências bibliográficas; qualidade da escrita e formatação do resumo e da introdução; clareza da definição dos objetivos; adequação da descrição da metodologia; clareza da apresentação dos resultados; 
fundamentação da discussão em trabalhos citados na lista de referências; clareza e coesão da conclusão com a proposição, os resultados obtidos e discussão apresentada.

Os títulos dos relatórios produzidos no ano de 2019 estão descritos na tabela 2. Vale ressaltar que nenhum desses relatórios ou de dados neles constantes foram ou serão utilizados para elaboração de TCC ou de qualquer trabalho de pesquisa.

Tabela 1. Conteúdo teórico, atividade prática e objetivo pedagógico das atividades realizadas

\begin{tabular}{|c|c|c|}
\hline Aula Teórica & Aula Prática & Objetivo Pedagógico \\
\hline Sistemas tampão & $\begin{array}{l}\mathrm{pH} \text { e capacidade tamponante } \\
\text { da saliva }\end{array}$ & $\begin{array}{l}\text { Correlacionar os parâmetros analisados com } \\
\text { a manutenção da integridade da estrutura } \\
\text { mineralizada dos dentes }\end{array}$ \\
\hline Enzimas & $\begin{array}{l}\text { Determinação da atividade da } \\
\text { fosfatase ácida na saliva }\end{array}$ & $\begin{array}{l}\text { Entender a importância das enzimas nos } \\
\text { processos metabólicos na saúde e na doença, } \\
\text { além de seus usos como biomarcadores } \\
\text { salivares de doenças bucais e sistêmicas }\end{array}$ \\
\hline Proteínas e peptídeos salivares & $\begin{array}{l}\text { Determinação do conteúdo de } \\
\text { proteína total na saliva }\end{array}$ & $\begin{array}{l}\text { Contextualizar a multifuncionalidade das } \\
\text { proteínas e peptídeos salivares na promoção } \\
\text { da saúde dos tecidos bucais (lubrificação, } \\
\text { ação tamponante, proteção, inibição da } \\
\text { formação de cálculo dentário etc.) }\end{array}$ \\
\hline $\begin{array}{lll}\text { Proteínas } & \text { do } & \text { tecido } \\
\text { mineralizado } & & \end{array}$ & $\begin{array}{l}\text { Determinação da atividade da } \\
\text { fosfatase alcalina na saliva }\end{array}$ & $\begin{array}{l}\text { Reforçar a importância das enzimas como } \\
\text { biomarcadores salivares do dano ao tecido } \\
\text { periodontal }\end{array}$ \\
\hline $\begin{array}{lcr}\text { Digestão e absorção dos } \\
\text { principais componentes da } \\
\text { dieta }\end{array}$ & $\begin{array}{l}\text { Determinação da atividade de } \\
\alpha \text {-amilase na saliva }\end{array}$ & $\begin{array}{l}\text { Identificar a importância da } \alpha \text {-amilase na } \\
\text { função digestiva e como biomarcador salivar } \\
\text { de estresse emocional e físico. }\end{array}$ \\
\hline $\begin{array}{l}\text { Redução parcial do oxigênio e } \\
\text { geração de espécies reativas de } \\
\text { oxigênio }\end{array}$ & $\begin{array}{l}\text { Determinação do ácido úrico } \\
\text { na saliva }\end{array}$ & $\begin{array}{l}\text { Entender a importância do ácido úrico como } \\
\text { componente da defesa antioxidante não- } \\
\text { enzimática salivar na proteção contra os } \\
\text { danos oxidativos }\end{array}$ \\
\hline Halitose & $\begin{array}{l}\text { Determinação da atividade da } \\
\text { transaminase na saliva }\end{array}$ & $\begin{array}{l}\text { Associar o aumento da atividade de enzimas } \\
\text { de metabolismo de aminoácidos à produção } \\
\text { de odorivetores associados à halitose }\end{array}$ \\
\hline $\begin{array}{l}\text { Efeitos metabólicos da } \\
\text { epinefrina, glucagon e insulina. } \\
\text { Regulação integrada do } \\
\text { metabolismo }\end{array}$ & $\begin{array}{l}\text { Determinação da glicose na } \\
\text { saliva }\end{array}$ & $\begin{array}{l}\text { Explorar a correlação entre os biomarcadores } \\
\text { salivares e as doenças sistêmicas, em } \\
\text { especial o diabetes }\end{array}$ \\
\hline $\begin{array}{l}\text { Composição mineral do dente e } \\
\text { da saliva }\end{array}$ & $\begin{array}{l}\text { Determinação de cálcio e } \\
\text { fósforo na saliva }\end{array}$ & $\begin{array}{l}\text { Aprender sobre a composição e a } \\
\text { importância da manutenção do equilíbrio } \\
\text { mineral entre a estrutura do dente e a saliva } \\
\text { na prevenção do processo de cárie dentária }\end{array}$ \\
\hline
\end{tabular}


Tabela 2. Títulos atribuídos pelos estudantes aos relatórios finais das atividades práticas da disciplina de Bioquímica no ano de 2019

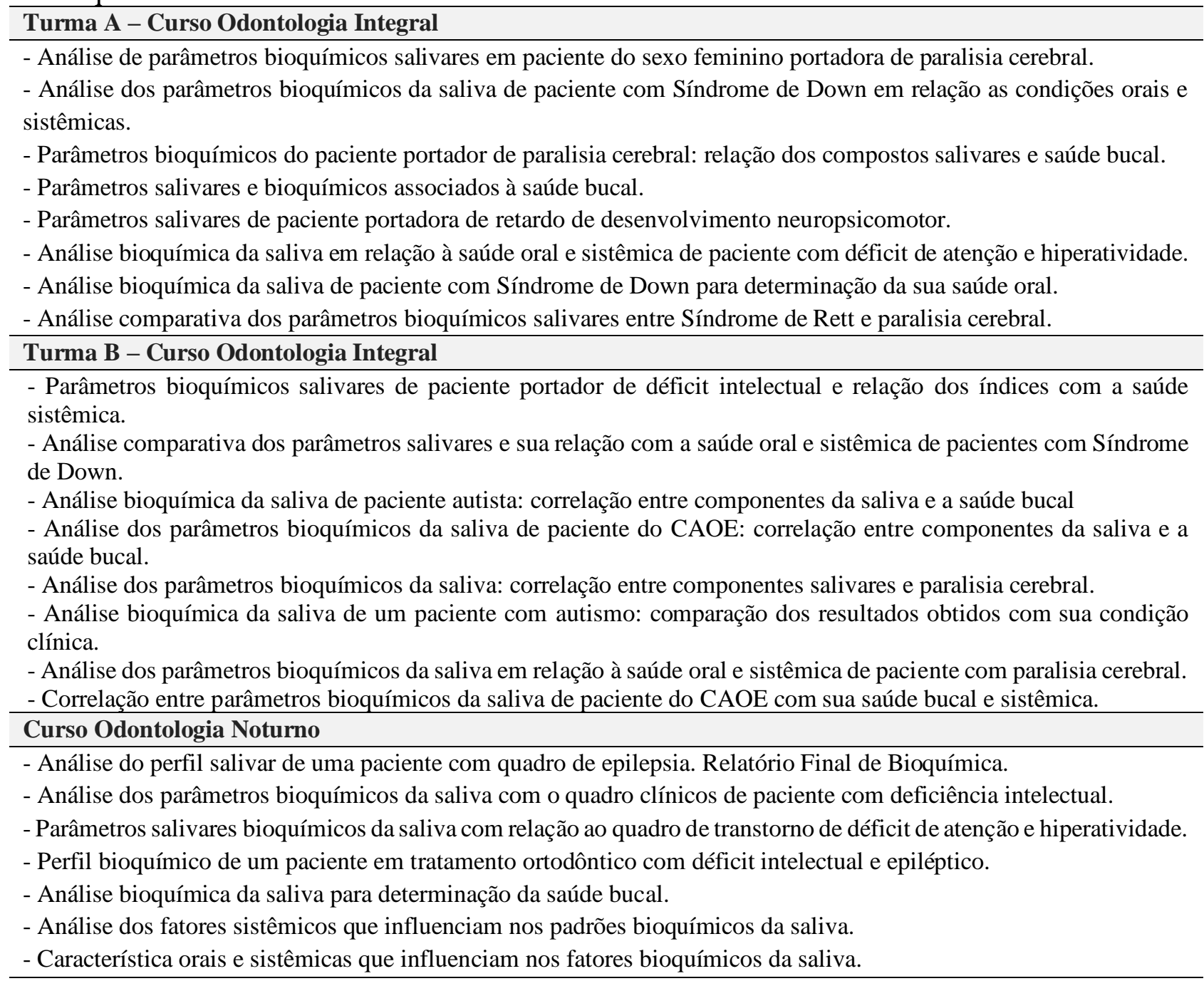

Na FOA-UNESP, o primeiro contato dos estudantes com atividades pré-clínicas ocorre no segundo ano da graduação, o que pode gerar certa frustação. Portanto, a atividade proposta e realizada pela disciplina de Bioquímica amenizou a expectativa, mantendo os estudantes motivados e, consequentemente, fortalecendo o interesse pelo curso, o que pôde ser observado ao longo do ano pelo baixíssimo número de ausências nas aulas práticas e o bom rendimento escolar das turmas, que resultou em elevada porcentagem de aprovação.
Considerando que a atividade foi realizada por um número expressivo de estudantes por turma, e estando bem estabelecido que o estilo de aprendizagem difere de indivíduo para indivíduo e é influenciado por experiências anteriores ${ }^{19}$, é natural que algumas deficiências tenham aflorado, como a dificuldade na leitura e compreensão dos artigos em inglês; a falta de noções de informática; as restrições para interpretar e seguir as normas de formatação do texto. A dificuldade para a interpretação dos procedimentos clínicos descritos no prontuário não pode ser incluída no citado 
acima, por ser inerente ao fato dos estudantes estarem cursando o $1^{\circ}$ ano da graduação.

No que se refere à dificuldade de interpretação de parâmetros bioquímicos salivares e sua associação à saúde bucal e sistêmica, esbarrou-se na ausência de valores de referência validados para a saliva, o que resulta, em certos casos, da inexistência de um consenso na literatura em relação às técnicas de coleta e processamento da saliva ${ }^{20,21}$. Apesar desses percalços, entende-se que não houve comprometimento suficiente para impedir o alcance dos objetivos propostos.

A motivação e o interesse pela Bioquímica devem ser, na medida do possível, mantidos pelos professores responsáveis ${ }^{22,23}$. Sendo assim, todos os conceitos foram expostos de maneira que as correlações com parâmetros clínicos sistêmicos e bucais dos pacientes ficassem evidenciadas, demostrando assim a aplicabilidade desses. Para nortear e auxiliar ainda mais os estudantes, ocorreram encontros com os professores e monitores da disciplina para a discussão de pontos específicos. Creditamos parte do sucesso desta atividade ao fato de terem sido estudados casos reais, de pacientes com os quais os estudantes tiveram contato direto ou indireto, o que os levou a um maior envolvimento que facilitou a aquisição, integração, discussão e a aplicação dos conceitos de Bioquímica.

As bases epistemológicas do ensino tradicional foram mantidas por meio de conteúdos preestabelecidos, transmissão unidirecional de conhecimento nas aulas teóricas e avaliação transversal. Contudo, a descrição do relato de experiência em questão demonstra a viabilidade da associação de um novo componente na estrutura da disciplina, por meio do desenvolvimento de atividades práticas nas quais o estudante é o sujeito ativo que conduz o processo de investigação científica, possibilitando aprimorar o conhecimento teórico e prático, proporcionando ações transformadoras em seu trajeto de formação profissional. Embora a análise do status epistemológico da Bioquímica indique problemas relacionados à combinação imaginária de entidades de química e biologia ${ }^{24}$, a curiosidade e o interesse do estudante sobre os conceitos abordados nas aulas teóricas foram estimulados ao integrarmos os resultados das análises salivares a um caso clínico real estudado.

O conjunto das atividades realizadas estimulou o domínio de aprendizagem cognitivo, uma vez que o desenvolvimento intelectual foi fomentado, e do domínio psicomotor, pois, na realização das dosagens laboratoriais, os estudantes tiveram que aprender a manusear vidrarias e equipamentos laboratoriais até então desconhecidos por eles.

\section{CONSIDERAÇÕES FINAIS}

Alguns fatores contribuem para o baixo desempenho e motivação para o estudo da Bioquímica. Dentre esses, destacam-se a abordagem superficial por parte dos estudantes no momento do estudo, privilegiando um processo mecânico de memorização, e a falta de conhecimento efetivo sobre a importância dos ensinamentos da disciplina em sua futura carreira profissional.

$\mathrm{Na}$ atividade proposta e aqui relatada, houve satisfatório envolvimento dos estudantes ao longo do ano letivo, maior valorização e compreensão da importância da Bioquímica, e entendimento da complexidade envolvida nos cuidados para com pacientes com necessidades especiais. Apesar de ansiarem por procedimentos práticos e clínicos, os estudantes demonstraram maior conscientização sobre a importância do conhecimento teórico essencial para a interpretação dos resultados obtidos, valorizando a importância da disciplina básica em sua formação.

Concluiu-se que a estratégia adotada é viável e pode beneficiar educadores que buscam alternativas que permitam a integração das ciências 
básicas e clínicas ao ensino de Bioquímica para a Odontologia.

\section{Agradecimentos}

A toda equipe do CAOE pela disponibilidade e cooperação em especial a Ana Lúcia Francischini Damaceno e as Professoras Letícia Helena Theodoro, Rita Cássia Menegati Dornelles e Alessandra Marcondes Aranega. Ao auxiliar acadêmico Ângelo Luiz Baiochi, responsável pelo preparo do laboratório multidisciplinar da FOA-UNESP e suporte técnico durante as aulas práticas. Aos monitores que auxiliaram não apenas durante as atividades práticas, como também nos plantões de dúvidas. Aos estudantes dos cursos de Odontologia Integral e Noturno da FOA-UNESP, pela perseverança na execução dos procedimentos experimentais e na elaboração dos relatórios. A Direção da FOAUNESP pelo apoio no fornecimento dos materiais consumíveis e kits comerciais. Agradecimento a Maria Rita Stevanato Nakamune pela revisão do português, inglês e espanhol.

\section{ABSTRACT \\ Biochemical analysis of the saliva of patients with cognitive and physical disabilities as a practical Biochemistry class strategy in the Dentistry course}

Given the importance of teaching biochemistry in dental surgeon training courses, the objective was to present a pedagogical strategy that enables the articulation of basic molecular concepts within the subject, allowing a better understanding of the complexity of oral and systemic diseases, through the biochemical analysis of saliva from patients with cognitive and physical disabilities at the Dental Assistance Center for Disabled Persons (CAOE) of the School of Dentistry, Araçatuba - São Paulo State University (UNESP). For this purpose, groups of five students analyzed the saliva collected by a dental surgeon from CAOE. Immediately after collection, salivary flow, $\mathrm{pH}$, and buffering capacity were determined. Subsequently, the saliva was centrifuged, fractionated, and stored at
$-20{ }^{\circ} \mathrm{C}$ until the time of biochemical analysis. During the practical classes, using commercial kits, the samples were tested for: total protein, $\alpha-$ amylase, alkaline phosphatase, uric acid, glucose, aspartate aminotransferase, calcium, and phosphorus. At the end of the school year, students presented a report contextualizing the results with the patient's oral and systemic health. Tutoring sessions with monitors and teachers helped in the interpretation of the anamnesis form and the correlations of oral and systemic clinical parameters. For incoming students, this was the first opportunity to integrate theoretical knowledge with clinical perspectives. It is concluded that the adopted strategy is viable and can benefit educators who seek alternatives that allow the integration of basic and clinical sciences for teaching Biochemistry in Dentistry. Descriptors: Learning. Teaching. Education, Dental. Biochemistry. Saliva.

\section{REFERÊNCIAS}

1. Guven Y. Scientific basis of dentistry. J Istanb Univ Fac Dent. 2017;51(3):64-71.

2. Humphrey SP, Mathews RE, Kaplan AL, Beeman CS. Undergraduate basic science preparation for dental school. J Dent Educ. 2002;66(11):1252-9.

3. Gadbury-Amyot CC, Overman PR, Crain G. The development and implementation of an online applied biochemistry bridge course for a dental hygiene curriculum. J Dent Educ. 2009;73(1):83-94.

4. Dabaghi-Tabriz F, Ghaffari R, Hanaee J, Barzegar M, Bilan N, Dehghani AH, et al. Comparison of General Dentistry Curriculum in Iran with Eight of the World's Top Dental Faculties. Maedica (Buchar). 2019;14(2):104-15.

5. Kulak V, Newton G. A guide to using casebased learning in biochemistry education. Biochem Mol Biol Educ. 2014;42(6):45773.

6. Hartfield PJ. Reinforcing constructivist 
teaching in advanced level Biochemistry through the introduction of case-based learning activities. J Learn Des. 2010;3(3):20-31.

7. Davis MC. The Successful Use of Case Studies in Biochemistry. Ga J Sci. 2004;62(2):79-86.

8. Biggs J, Tang C. Teaching for Quality Learning at University: What the Student Does. 4th ed. Berkshire: The Society for Research into Higher Education \& Open University Press; 2011.

9. Brasil. Ministério da Educação. Conselho Nacional de Educação. Câmara de Educação Superior. Resolução CNE/CES nº 3/2002, de 19 de fevereiro e 2002 [Institui Diretrizes Curriculares Nacionais do Curso de Graduação em Odontologia]. Diário Oficial da República Federativa do Brasil. 2002, 04 de mar; Seção 1:10.

10. Formicola AJ. Preprofessional preparation for the basic science curriculum. J Dent Educ. 1986;50(8):458-61.

11. Graham R, Bitzer LA, Anderson OR, Klyvert M, Moss-Salentijn L, Lamster IB. Advancing the educational training of dental educators: review of a model program. J Dent Educ. 2012;76(3):303-10.

12. Jesus JTA, Santos JÁ, Conceição MP, Silva TR, Gonçalves NO, Yarid SD. Primeiro contato do discente com a clínica: relato de experiência. Rev ABENO. 2016;16(3):7884.

13. Khanova J, McLaughlin JE, Rhoney DH, Roth MT, Harris S. Student Perceptions of a Flipped Pharmacotherapy Course. Am J Pharm Educ. 2015;79(9):140.

14. Neves BS, Marcelo IO, Naumann MH, Mello-Carpes PB. Ciências Básicas através de Casos Clínicos: Percepção dos Estudantes de Fisiologia sobre o Uso deste Método. Rev Ens Bioq. 2019;17.
15. Carvalho EMC, Araújo RPC. A Saúde Bucal em portadores de transtornos mentais e comportamentais. Pesqui Bras Odontopediatria Clín Integr. 2004;4(1):6575.

16. Tibell LA, Rundgren CJ. Educational challenges of molecular life science: Characteristics and implications for education and research. CBE Life Sci Educ. 2010;9(1):25-33.

17. Tian Z, Zhang K, Zhang T, Dai X, Lin J. Application of Ausubel cognitive assimilation theory in teaching/learning medical biochemistry and molecular biology. Biochem Mol Biol Educ. 2019;118.

18. Psillos D, Niedderer SM. Teaching and Learning in the Science Laboratory. 1st ed. New York: Kluwer Academic Publishers; 2002.

19. Silva MEF, Galembeck E. Preferências de Estilos de Aprendizagem entre os usuários da Biblioteca Digital de Ciências (BDC-IBUnicamp). Rev Bras Pesqui Educ Cienc. 2014;14(1):171-89.

20. Celec P, Ostatnikova D. Saliva collection devices affect sex steroid concentrations. Clin Chim Acta. 2012;413(19-20):1625-8.

21. Golatowski C, Salazar MG, Dhople VM, Hammer E, Kocher T, Jehmlich N, et al. Comparative evaluation of saliva collection methods for proteome analysis. Clin Chim Acta. 2013;419:42-6.

22. Oliveira RC, Iano FG, Silva TL, Buzalaf MAR. Percepção dos alunos do curso de odontologia de uma universidade brasileira em relação à importância da disciplina de bioquímica na sua profisssão. Rev Bras Ens Bioq Biol Mol. 2007(1):B1-B6.

23. Oliveira RC, Monteiro NFG, Iano FG, Silva TL, Buzalaf MAR. Expectativas dos alunos quanto à importância da bioquímica em sua 
carreira. Rev Bras Ens Bioq Biol Mol. Correspondência para: 2010(1):D1-D9.

Ana Cláudia de Melo Stevanato Nakamune

24. Jacob C. Philosophy and Biochemistry: Research at the Interface between Chemistry and Biology. Found Chem.2002(4):97-125.

e-mail: ana.nakamune@unesp.br

Faculdade de Odontologia de Araçatuba

FOA-UNESP

Departamento de Ciências Básicas

Rodovia Marechal Rondon 527/528 km

Câmpus Universitário S/N

16018-805 Araçatuba/SP 